\title{
Mastectomía contralateral profiláctica con reconstrucción inmediata en pacientes con cáncer de mama unilateral sin mutación germinal en BRCA1/2
}

\author{
Mara Cruellas Lapeña ${ }^{a, *}$, Ismael Gil Romea ${ }^{b}$, Luis Mariano Esteban ${ }^{c}$, \\ Carlos Álvarez Ruiz ${ }^{c}$, Natalia Alonso Marin ${ }^{a}$, Maitane Ocariz Diez ${ }^{a}$, \\ Andrea Sesma Goñi ${ }^{a}$, Marta Gascón Ruiz ${ }^{a}$, María Zapata García ${ }^{a}$, \\ María Zurera Berjaga ${ }^{a}$, Elisa Quilez Bielsa ${ }^{a}$, María Álvarez Alejandro ${ }^{a}$, \\ Irene Torres Ramón ${ }^{a}$, Pablo Andrés Ordóñez Lozano ${ }^{d}$, Pilar Bueso Inglán ${ }^{a}$, \\ Dolores Isla Casado ${ }^{a}$ y Raquel Andrés Conejero ${ }^{a}$
}

\footnotetext{
a Oncología médica, Hospital Clínico Universitario Lozano Blesa, Zaragoza, España

b Cirugía General, Hospital Clínico Universitario Lozano Blesa, Zaragoza, España

c Escuela Politécnica La Almunia, Universidad de Zaragoza, La Almunia de Doña Godina, Zaragoza, España

d Cirugía torácica, Hospital MAZ, Zaragoza, España
}

Recibido el 11 de abril de 2020; aceptado el 13 de julio de 2020

\section{PALABRAS CLAVE \\ Cáncer de mama; \\ Cirugía mamaria; \\ Mastectomía \\ contralateral}

\begin{abstract}
Resumen
Introducción: No existe un consenso sobre las indicaciones de mastectomía contralateral en pacientes diagnosticadas de cáncer de mama unilateral sin mutación germinal en BRCA1/2. Estudios previos han identificado algunos factores que pueden influir en la toma de la decisión dependientes del tumor, como el tamaño o histología, de la paciente, como la edad, y de la cirugía como la posibilidad de realizar una reconstrucción inmediata o la experiencia del cirujano.

Métodos: Estudio retrospectivo de una cohorte de 176 pacientes diagnosticadas de CM entre 2010 y 2016 a las que se les realizó cirugía mamaria. Se ha analizado la asociación de características del tumor y de la paciente con la toma de decisión de realizar mastectomía contralateral (MC) o no-MC. Asimismo, se han analizado los datos relacionados con la cirugía y la recurrencia por grupos mediante la curva de incidencia acumulada y el test de Gray.

Resultados: El número de MC se ha incrementado en nuestro centro. No hemos encontrado diferencias significativas en el desarrollo de complicaciones posquirúrgicas entre los 2 grupos de pacientes, pero sí en la estancia hospitalaria, siendo superior para MC. También hemos observado diferencias entre ambas cohortes en edad y tipo de tumor, siendo la MC más frecuente en aquellas pacientes más jóvenes y subtipo luminal $A$. Hemos hallado diferencias en la incidencia acumulada de recidiva entre ambos subgrupos $(p=0,034)$.
\end{abstract}

Abreviaturas: CAP, complejo areola-pezón; CM, cáncer de mama; CMC, cáncer de mama contralateral; MC, mastectomía contralateral. * Autor para correspondencia.

Correo electrónico: mara1290@gmail.com (M. Cruellas Lapeña). 
Conclusiones: En nuestra cohorte la MC se realiza más frecuentemente en pacientes más jóvenes y con cáncer de mama luminal $A$.

(C) 2020 Publicado por Elsevier España, S.L.U. en nombre de SESPM.

\section{KEYWORDS}

Breast cancer;

Breast surgery;

Contralateral

mastectomy

\section{Introducción}

La MC consiste en la exéresis de la mama sana contralateral en aquellas mujeres diagnosticadas de cáncer de mama unilateral para reducir el riesgo desarrollar un cáncer de mama contralateral (CMC). El riesgo de desarrollo de CMC es de $0,5-1 \%$ por año ${ }^{1,2}$, dependiendo de factores como la edad, la historia familiar o el tratamiento hormonal adyuvante. La MC se ofrece en aquellas pacientes portadoras de mutación germinal en $B R C A 1 / 2$ por su riesgo incrementado de $\mathrm{CMC}^{2-4}$. Las tasas de realización de $M C$ se han incrementado a lo largo de los años ${ }^{3,5-7}$. Sin embargo, no queda claro en la literatura el impacto que dicho procedimiento tenga en supervivencia global o específica por cáncer de mama $^{7-12}$. El aumento de MC es evidente sobre todo en Estados Unidos, y varios factores se han relacionado con este incremento. Estos factores incluyen tanto la edad y características del tumor como el tamaño o el tipo y el grado histológico ${ }^{1,7,11-15}$. También se ha considerado la influencia del estatus socioeconómico ${ }^{1,7}$ que condiciona, en algunos países, el acceso a la técnica en dependencia del seguro contratado. En nuestra sociedad disponemos de un sistema de salud público universal, por lo que el acceso a las diferentes opciones terapéuticas no está determinado por el nivel económico.

El objetivo de este trabajo es analizar en nuestro medio si esta técnica se ha incrementado a lo largo de los años, qué factores pueden influir en la toma de decisiones y el impacto en supervivencia libre de recidiva.

\section{Material y métodos}

\section{Diseño}

Se trata un estudio retrospectivo unicéntrico longitudinal en el Hospital Clínico Universitario Lozano Blesa de Zaragoza, que incluyó a 176 pacientes intervenidas de cáncer de mama entre enero de 2010 y septiembre de 2016.

\section{Población a estudio y variables analizadas}

Se incluyeron aquellas pacientes intervenidas durante dicho periodo de cáncer de mama unilateral, con edad igual o inferior a 60 años y con un mínimo seguimiento de 6 meses. Se excluyeron aquellas pacientes diagnosticadas de carcinoma lobulillar in situ y aquellas diagnosticadas de cáncer de mama bilateral sincrónico. Se excluyeron las pacientes portadoras de mutación germinal en BRCA1 y BRCA2. No fue un criterio de exclusión una historia familiar positiva de cáncer de mama u ovario.

Se definieron 2 grupos de pacientes: pacientes a las que se había realizado $M C$ vs. a las que no. Las pacientes del grupo no-MC podían haber recibido los siguientes 
tratamientos quirúrgicos: cirugía conservadora, mastectomía unilateral y cirugía bilateral de simetrización. En todas las pacientes del grupo MC se llevó a cabo la reconstrucción mamaria inmediata con implantes, y dependiendo del tipo de mama se adoptaba un tipo u otro de patrón de mastectomía en dependencia de las características morfológicas de cada mama. En el caso de mamas pequeñas se realizaron patrones laterales sin extirpación del complejo areola-pezón (CAP), quedando un remanente que se estima en un $4 \%$. En cambio, si las mamas eran voluminosas y ptosicas, las técnicas realizadas fueron de tipo SPIRA, en las que el CAP fue desinsertado e injertado posteriormente como un injerto libre. En los casos que se decidió por mastectomías tipo SPIRA no se utilizaron mallas para realizar el bolsillo protésico, utilizando colgajos dermograsos solos o unidos al músculo pectoral mayor una vez desinsertado de sus inserciones inferiores.

Se recogió la edad de las pacientes, el tipo de cirugía realizada, la estancia postoperatoria, si precisaron o no reintervención quirúrgica y la estancia hospitalaria derivada. También aquellas complicaciones que requirieron ingreso hospitalario y la duración.

De cada paciente se revisaron los tratamientos complementarios recibidos (hormonoterapia, radioterapia y/o quimioterapia). Referente a la quimioterapia se revisó si esta había sido con intención neoadyuvante o adyuvante, el esquema y número de ciclos recibidos. Asimismo, si alguna paciente había desarrollado alguna complicación por el tratamiento con quimioterapia que precisara ingreso hospitalario y su duración. En cuanto a lo referente a las características tumorales se recogieron el estadio tumoral por el sistema TNM, el tipo histológico y el subtipo de cáncer de mama, y también los hallazgos preneoplásicos o neoplásicos en el estudio anatomopatológico de la mama contralateral en aquellas pacientes a las que se les realizó MC. Además, se revisaron todas las pruebas complementarias que se habían realizado durante el seguimiento. El tiempo de seguimiento se calculó entre la fecha de la última consulta en la unidad de mama o exitus y la fecha de diagnóstico.

El estudio fue aprobado por la comisión de ética previo al inicio de la recogida de datos.

\section{Análisis estadístico}

Las variables clínicas fueron analizadas de forma descriptiva. Se recogieron varias variables clínicas, así como el diagnóstico posterior de recidiva locorregional y cáncer de mama contralateral. Para el análisis comparado de recurrencia por grupos de $M C /$ no-MC, el tiempo de seguimiento se calculó desde la fecha de diagnóstico hasta la fecha de recurrencia, considerada como la aparición de la enfermedad a nivel locorregional o cáncer de mama contralateral, o la última visita en el grupo de pacientes que no experimentaron recurrencia.

En el estudio univariante comparativo entre los subgrupos las variables continuas fueron analizadas con el test de Shapiro-Wilk, y no se verificó la normalidad, por lo que se han descrito con medianas y rangos intercuartílicos, y las diferencias significativas entre grupos fueron analizadas con el test de MannWithney. Las variables categóricas fueron
Tabla 1 Proporción de MC del total de cirugías mamarias por cáncer de mama realizadas

\begin{tabular}{ll}
\hline Año & Proporción $(M C /$ total $\times 100)$ \\
\hline 2010 & $24 \%$ \\
2011 & $26,92 \%$ \\
2012 & $42,3 \%$ \\
2013 & $34,78 \%$ \\
2014 & $63,15 \%$ \\
2015 & $48 \%$ \\
\hline
\end{tabular}

No se ha incluido 2016 por no haber recogido los datos completos de ese año.

descritas con frecuencias relativas y absolutas y comparadas con el test de Chi-cuadrado. El análisis de recurrencia fue estudiado con las curvas de incidencia acumulada y el test de Gray para la comparación entre grupos MC/no-MC. Los datos fueron analizados usando el lenguaje de programación $R$ versión 3.6.1 (The R Foundation for Statistical Computing, Viena, Austria).

\section{Resultados}

La edad mediana de las pacientes fue de 48 (43-53) años. De las 176 pacientes intervenidas a 68 se les realizó MC $(38,63 \%)$. La estancia mediana posquirúrgica fue de 6 días (4-8 días) (7 días de mediana para el grupo de MC vs. 5 días de mediana para el grupo de no-MC). Sesenta y tres pacientes $(35,79 \%)$ precisaron reintervención quirúrgica posterior (16 pacientes en el grupo de MC vs. 47 pacientes en el grupo no-MC). El 47,61\% de las reintervenciones fueron debidas a extracción/recambio/colocación de expansor/prótesis. En el grupo no-MC se incluía a aquellas pacientes a las que se les había realizado tanto cirugía conservadora como mastectomía o cirugía de simetrización con la mama contralateral. Un total de 23 pacientes (13,06\%) desarrollaron complicaciones relacionadas con la cirugía (6 pacientes en el grupo de $M C$ vs. 17 pacientes en el grupo de no-MC). La histología de la mayoría de tumores analizados correspondía a un carcinoma ductal infiltrante $(77,2 \%)$; el estadio al diagnóstico de la mayoría de pacientes fue estadio II $(43,9 \%)$. El subtipo luminal B fue predominante $(52,6 \%)$ en el total de la cohorte estudiada y para ambos subgrupos. El 79,54\% de las pacientes recibió tratamiento complementario con quimioterapia; el $28,97 \%$ con intención neoadyuvante y el $50 \%$ restante como tratamiento adyuvante. De las 176 pacientes incluidas 8 fueron diagnosticadas de recidiva locorregional durante el seguimiento ( 2 pacientes en el grupo de MC y 6 pacientes en el grupo no-MC) y 8 pacientes del subgrupo no-MC desarrollaron con posterioridad un cáncer de mama contralateral. También se analizaron el número de hallazgos ocultos de la mama contralateral en aquellas pacientes en las que se realizó MC. Tras el estudio anatomopatológico en un $5 \%(n=9)$ de las pacientes se observó hiperplasia ductal atípica o carcinoma lobulillar in situ.

Si comparamos el número de MC llevadas a cabo entre el año 2010 y 2016 observamos que el porcentaje de MC realizadas se ha incrementado (tabla 1 y fig. 1).

En el estudio comparativo entre subgrupos se hallaron diferencias estadísticamente significativas en la edad, 


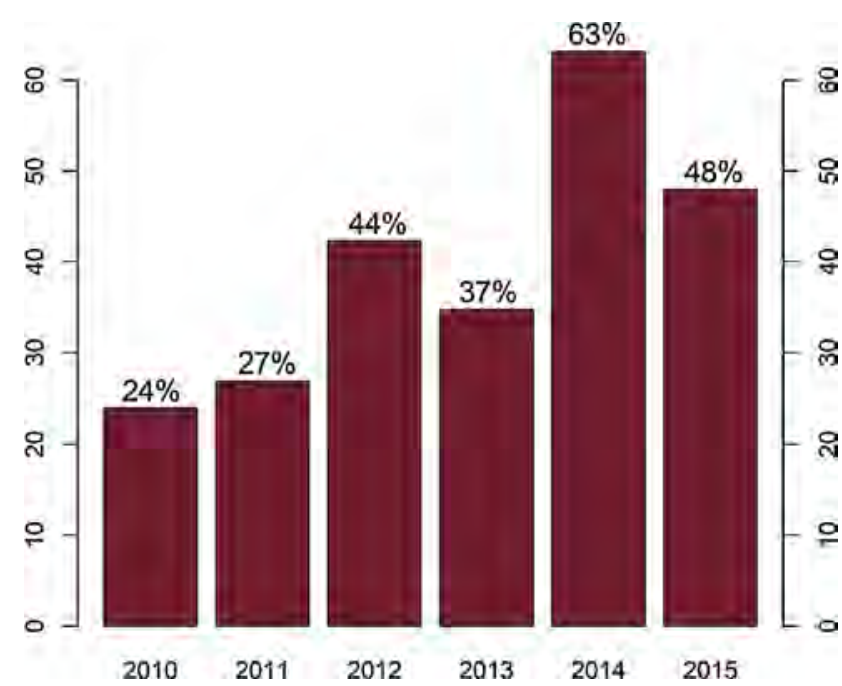

Figura 1 Proporción de MC del total de cirugías mamarias por cáncer de mama realizadas.

No se ha incluido 2016 por no haber recogido los datos completos de ese año.

siendo más jóvenes las del grupo de MC (MC: 47 [40-51] años vs. no-MC: 50 [44-54] años; $\mathrm{p} \leq 0,006$ ). También se hallaron diferencias estadísticamente significativas en cuanto a los días de estancia postquirúrgica (MC: 7 días vs. no-MC: 5 días; $p \leq 0,001$ ), número de reintervenciones precisadas (MC: $23,52 \%$ vs. no-MC: $43,51 \% \mathrm{p}=0,011$ ) y el número de días de ingreso por la reintervención (MC: 3 días vs. no-MC: 5 días; $p \leq 0,001)$. Asimismo, se observaron diferencias significativas en el subtipo de cáncer de mama entre ambos subgrupos $(p=0,004)$, siendo más frecuente el subtipo Luminal $A$ en el grupo $M C$ ( $M C$ : 35,3\% vs. no-MC: $15,2 \% ; p=0,003$ ) (tabla 2). En el subtipo luminal B se han observado asimismo diferencias significativas, siendo más frecuente la no-MC en dicho subgrupo. No se observaron diferencias entre ambos subgrupos en las complicaciones posquirúrgicas, estadio o histología.

Tras realizar el análisis multivariante en el que se incluye la edad, la histología, el estadio y el subtipo, la significación estadística se mantiene para la edad y el subtipo luminal $A$ $(p=0,004$ y $p=0,0004)$

En cuanto al seguimiento de las pacientes la mediana fue de 48 meses (rango intercuartílico 32-66 meses), con una mediana de tiempo hasta la recidiva de 27 meses (rango intercuartílico 20-37 meses). Se hallaron diferencias significativas en la incidencia acumulada de recurrencia locorregional o contralateral entre ambos subgrupos (Gray test $p=0,034)$. En la figura 2 se muestra la curva de

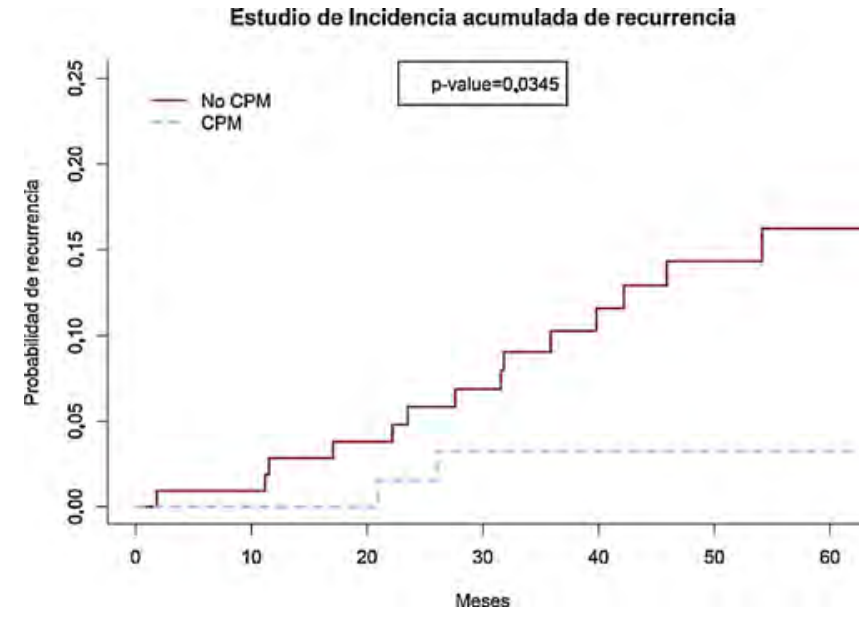

Figura 2 [vs1] Curva de incidencia acumulada de la recurrencia, que representa la probabilidad de recurrencia.

$0=$ no $-M C ; 1=M C$.

incidencia acumulada de recurrencia, donde se puede apreciar la diferencia en la probabilidad de recurrencia entre ambos subgrupos.

\section{Discusión}

El incremento de la realización de $M C$ es un hecho claramente establecido a nivel global. Sin embargo, no están claramente establecidos los criterios de realización de $M C$ en aquellas pacientes sin mutación germinal en BRCA1 o $B R C A$ 2.

Con este estudio se han analizado determinados factores que pueden influir en la toma de dicha decisión.

En cuanto a factores quirúrgicos la realización de la $M C$ junto con la reconstrucción inmediata, técnica de elección en nuestro centro, puede influir en la elección de la opción quirúrgica ${ }^{7}$. Algunas pacientes prefieren dicha cirugía para asegurar una mayor simetría mamaria ${ }^{16}$. Se ha analizado si existen diferencias en cuanto a la estancia postoperatoria o desarrollo de complicaciones posquirúrgicas. La cirugía profiláctica contralateral conlleva mayor agresión quirúrgica y mayor tiempo quirúrgico, lo que puede implicar mayor probabilidad de desarrollo de complicaciones posquirúrgicas. Sin embargo, en nuestra cohorte no hemos hallado diferencias significativas entre ambos subgrupos, dato que coincide con lo descrito en otros estudios ${ }^{17-19}$. En nuestra cohorte la estancia posquirúrgica tras la primera intervención es significativamente superior en las pacientes del grupo $M C$ con respecto a las del grupo no-MC, lo que sí que puede atribuirse

Tabla 2 Estudio comparativo entre ambos subgrupos en los que se han objetivado diferencias significativas

\begin{tabular}{lllll}
\hline & Total $(\mathrm{n}=176)$ & $M C(\mathrm{n}=68)$ & No $M C(\mathrm{n}=108)$ & Valor de $\mathrm{p}$ \\
\hline Edad & $48(43-53)$ & $47(40-51)$ & $50(44-54)$ & 0,006 \\
Días estancia tras operación & $6(4-8)$ & $7(6-9)$ & $5(3-7)$ & $<0,001$ \\
Número de reintervenciones & $63(35,79 \%)$ & $16(23,52 \%)$ & $47(43,51 \%)$ & 0,011 \\
Luminal A & $40(23,1 \%)$ & $24(35,3 \%)$ & $16(15,2 \%)$ & 0,003 \\
Luminal B & $91(52,6 \%)$ & $25(36,8 \%)$ & $66(62,9 \%)$ & 0,003 \\
\hline
\end{tabular}


a la mayor complejidad de dicha técnica. Sin embargo, el número de reintervenciones fue significativamente superior en el grupo de no-MC. Esto bien ha podido deberse a la reconstrucción inmediata realizada en la $M C$, lo que evitaría potencialmente cirugías reconstructivas posteriores. También se han incluido en dichas reintervenciones las linfadenectomías, la ampliación de márgenes y en algunos casos cirugías de simetrización para conseguir mejores resultados estéticos o cirugía bilateral profiláctica realizada con posterioridad.

Referente al tipo de cirugía, en la mayoría de estudios se divide a aquellas pacientes en 2 subgrupos, uno en el que se realiza $M C$ y otro en el que no, sin detallar en profundidad el tipo de cirugía, aunque sí especificando en ocasiones si iba o no acompañada de reconstrucción inmediata, como concreta nuestro estudio. En el estudio de Chen et al. define el subgrupo MC profiláctica como mastectomía ipsilateral con mastectomía contralateral profiláctica, y especifica un incremento del uso de reconstrucción con implantes ${ }^{11}$. Billing et al. seleccionan a aquellas pacientes diagnosticadas de CM unilateral a las que se les realiza reconstrucción mamaria inmediata, diferenciándolas posteriormente en 2 subgrupos: aquellas a las que se les realiza mastectomía unilateral con reconstrucción inmediata y aquellas a las que se les realiza mastectomía unilateral con mastectomía contralateral profiláctica y reconstrucción inmediata ${ }^{17}$. Osman et al. diferencian entre un subgrupo al que se le realizó mastectomía unilateral + biopsia selectiva de ganglio centinela vs. otro subgrupo al que se realizó mastectomía bilateral + biopsia selectiva de ganglio centinela ${ }^{16}$. Disa et al. subdividen a las pacientes entre aquellas en las que se llevó a cabo una mastectomía unilateral vs. aquellas en las que se realizó mastectomía unilateral junto con MC profiláctica. Además, especificaron que incluyeron tanto a las pacientes en las que se utilizó reconstrucción con tejido autólogo como con implante ${ }^{19}$.

Entre los factores diferenciales de ambas cohortes encontramos la edad, siendo más frecuente la $M C$ en pacientes más jóvenes. Este factor ha sido relacionado en múltiples estudios como factor influyente en la elección de $M C^{11,13}$.

Entre los factores relacionados con el tumor la $M C$ es significativamente más frecuente en el subtipo luminal A, a pesar de ser el subgrupo de mejor pronóstico asociado. Habría que valorar de forma individualizada qué otros factores han podido influir en dicha decisión, como el tamaño tumoral o la afectación ganglionar. Por ello, se realizó el estudio multivariante que siguió mostrando diferencias significativas tanto para la edad como para el subtipo luminal A. La explicación de dichos resultados podría ser el intentar evitar los segundos tumores en aquellas pacientes más jóvenes, con mayor tiempo estimado de vida de desarrollo de segundos tumores y de subtipo menos agresivo, en las cuales es más probable conseguir una curación.

De nuestra cohorte aproximadamente en el $5 \%$ de las pacientes a las que se realizó $M C$ se hallaron hiperplasia ductal atípica o carcinoma lobulillar in situ en la mama contralateral.

En un estudio retrospectivo, publicado por Nomikos et al., se describe que la incidencia de enfermedad mamaria es significativamente mayor en aquellas pacientes en las que se realiza mastectomía profiláctica, apoyando la necesidad de examen histológico para el hallazgo de lesiones precursoras o cáncer oculto ${ }^{20}$.

En nuestra cohorte se ha demostrado diferencias en la incidencia acumulada de recurrencia locorregional o contralateral entre ambos subgrupos, pero no se ha analizado la incidencia acumulada de mortalidad por el diseño del estudio. Dicho dato, hasta el momento en controversia, sería uno de los factores más importantes que podrían establecer o no la indicación de MC en determinados supuestos.

Entre las limitaciones del estudio destacamos que se trata de un estudio unicéntrico y retrospectivo. Al ser unicéntrico los resultados pueden verse afectados por la experiencia quirúrgica en MC del equipo de cirugía. Además, no se ha recogido la historia familiar, la densidad mamaria, ni el estado de ansiedad/angustia de la paciente secundarias al diagnóstico, factores que pueden influir en la toma de decisiones por parte del facultativo y de la paciente.

\section{Conclusiones}

Tras el análisis multivariante se observó que en nuestra cohorte la MC se realiza más frecuentemente en pacientes más jóvenes y con cáncer de mama luminal $A$.

\section{Conflicto de intereses}

Los autores declaran no tener ningún conflicto de intereses.

\section{Bibliografía}

1. Chagpar AB. Prophylactic bilateral mastectomy and contralateral prophylactic mastectomy. Surg Oncol Clin N Am [Internet]. 2014;23:423-30.

2. Boughey JC, Attai DJ, Chen SL, Cody HS, Dietz JR, Feldman SM, et al. Contralateral prophylactic mastectomy (CPM) Consensus Statement from the American Society of Breast Surgeons?: Data on CPM outcomes and risks. Ann Surg Oncol. 2016;23:3100-5.

3. Wong SM, Freedman RA, Sagara Y, Aydogan F, Barry WT, Golshan M. Growing use of contralateral prophylactic mastectomy despite no improvement in long-term survival for invasive breast cancer. Ann Surg. 2017;265:581-9.

4. Ayala de la Peña F, Andrés R, Garcia-Sáenz JA, Manso L, Margelí M, Dalmau E, et al. SEOM clinical guidelines in early stage breast cancer. Clin Transl Oncol. 2019;21:18-30.

5. Kushary D, Suplee PD. Rising rates of contralateral prophylactic mastectomy as a treatment for early-stage breast cancer. Cancer Nurs. 2019;42:12-9.

6. Tuttle TM, Habermann EB, Grund EH, Morris TJ, Virnig BA. Increasing use of contralateral prophylactic mastectomy for breast cancer patients: a trend toward more aggressive surgical treatment. J Clin Oncol. 2007;25:5203-9.

7. Benson JR, Winters ZE. Contralateral prophylactic mastectomy. Br J Surg. 2016;103:1249-50.

8. Bedrosian I, Hu CY, Chang GJ. Population-based study of contralateral prophylactic mastectomy and survival outcomes of breast cancer patients. J Natl Cancer Inst. 2010;102: 401-9.

9. Bjelic-Radisic V, Singer C, Tamussino K, Kölbl H, Petru E, Volgger $\mathrm{B}$, et al. Contralateral prophylactic mastectomy in women with breast cancer without a family history or genetic predisposition: Consensus statement from the Austrian Gynecologic Oncology Working Group of the Austrian Society of Obstetrics and Gynecology. Wien Klin Wochenschr. 2019;131:233-6. 
10. Jatoi I, Parsons HM. Contralateral prophylactic mastectomy and its association with reduced mortality: Evidence for selection bias. Breast Cancer Res Treat. 2014;148:389-96.

11. Chen H, Zhang P, Zhang M, Wang M, Bai F, Wu K. Growing trends of contralateral prophylactic mastectomy and reconstruction in young breast cancer. J Surg Res. 2019;239: 224-32.

12. Lazow SP, Riba L, Alapati A, James TA. Comparison of breastconserving therapy vs mastectomy in women under age 40: National trends and potential survival implications. Breast J. 2019;25:578-84.

13. Metcalfe KA, Retrouvey H, Kerrebijn I, Butler K, O’Neill AC, Cil $\mathrm{T}$, et al. Predictors of uptake of contralateral prophylactic mastectomy in women with nonhereditary breast cancer. Cancer. 2019;125:3966-73.

14. Hegde JV, Wang X, Attai DJ, DiNome ML, Kusske A, Hoyt AC, et al. Assessing the effect of lifetime contralateral breast cancer risk on the selection of contralateral prophylactic mastectomy for unilateral breast cancer. Clin Breast Cancer. 2018;18:205-18.

15. Keskey RC, LaJoie AS, Sutton BS, Kim IK, Cheadle WG, McMasters $\mathrm{KM}$, et al. Cost-effectiveness analysis of contralateral prophylactic mastectomy compared to unilateral mastectomy with routine surveillance for unilateral sporadic breast cancer. Ann Surg Oncol. 2017;24:3903-10.

16. Osman F, Saleh F, Jackson TD. Increased postoperative complications in bilateral mastectomy patients compared to unilateral mastectomy: An analysis of the NSQIP database. Ann Surg Oncol. 2013:3212-7.

17. Billig JI, Duncan A, Zhong L, Aliu O, Sears ED, Chung KC, et al. The cost of contralateral prophylactic mastectomy in women with unilateral breast cancer. Plast Reconstr Surg. 2018;141:1094-102.

18. Alba B, Schultz BD, Cohen D, Qin AL, Chan W, Tanna N. Risk-tobenefit relationship of contralateral prophylactic mastectomy: The argument for bilateral mastectomies with immediate reconstruction. Plast Reconstr Surg. 2019;144:1-9.

19. Disa JJ, Matros E. Discussion: Risk-to-Benefit Relationship of Contralateral Prophylactic Mastectomy: The Argument for Bilateral Mastectomies with Immediate Reconstruction. Plast Reconstr Surg. 2019;144:10-1.

20. Nomikos A, Husain EA, Graham AD. Occult disease in reduction mammoplasties and prophylactic mastectomies. Breast J. 2019:1-6. 